\title{
Specialized channels for cognitive responses
}

\author{
M. JEANNEROD* \\ Laboratoire de Neuropsychologie \\ Expérimentale
}

In this note, I will try to show that there are several modes of response to external events, and that cognitive responses, from which cognition is inferred, is one. Also, I will advance the hypothesis that responses to external events are mediated by separate neural channels each of which is characterized by a given input-output relationship and thence by a given production. As an example, in visuomotor behavior the different properties of visual objects (e.g., shape, color, spatial location) are not processed by the same neural structures. They are matched by specifis mechanisms which generate motor commands appropriate for each property (for a more complete version of the hypothesis, see Jeannerod, 1981).

Now the question is this: are there cognitive char nels? By this I mean co channels exist that deal with the cognitive aspect of a situation $i$ and produce cognitive responses? A visual object for instance may be subjected to different levels of processing, besides that effected by visuomotor channels. Prociuction of a verbal response, mental representation, compa ison with other objects, etc., are based on the same object properties as tho e feeding into visuomotor structures. Can they be conceived as resulting from the activity of other parallel, specialized neural mechanisms?

My arguments are drawn from the study of brain-lesioned subjects. Over the last nundred years neurological observation has accumulated a considerable amount of information that seems to have been largely neglected by people involved in cognitive science. It is good news to hear that they have finally di covered this ignored continent (Posner et al., 1981).

Subjects with bilateral lesions of the visual cortex of the brain may displiy the typical syndrome of cortical blindness, once called 'psychic' blindness. They do not see, by the conventional meaning of the word 'see' since they do not experience visual changes or events. Some of them may even be unaware of not seeing, and may report hallucinatory visual scenes unrelated to the on-going physical reality. Yet, in cortical blindness, only part of the visual system is actually altered by the lesion. Other pathways also originating in the retina but terminating in areas outside the visual cortex are anatomi-

\footnotetext{
*Reprint reques:s shculd be addressed to $M$. Jeannerod, Laboratnire de Neuropsychologie Expérimentale, INSERM U94, 69500 Bro?, France.
} 
cally intact. As a matter of fact, visual responses such as eye movements to bright moving objects can still be elicited from these patients, although they remain unaware of the stimulus. Thus, in the case of psychic blindness only a certain mode of neural processing can be effected up to the point where it leads to an appropriate response. This response reflects the activity of surviving structures. The cortical lesion cuts off the access of visual information to the 'mental organ' specialized for cognitive operations, although the same information remains available to the visuomotor organ. According to the channel hypothesis the visuumotor organ only does what it is built fol, that is, it extracts a limited number of parameters from the risual workd and produces the corresponding responses.

A more dramatic illustration of this point is given by another group of pacients with more localized lesions of the visual cortex. In the case of unilateral lesions, cortical blindness is limited to a particular area of the visual field (the scotoma) in the half field contralateral to the lesion. It has recently been observed that the scotoma is only a 'relatively' blind area, from where responses to visual stimulation can still be obtained. The important point is that whether a response can be obtained or not depends on the requirements of the task. If the task requires a verbal response based on subjective experience of the stimulus (using the common perceptual or cognitive $2 \mathrm{~d} \%$ ), no response is given. If, on the other hand, the subject is forced to 'reach' (by eye or by hand), f $z$ a stimulus briefly presented within the scotoma a clear visuomotor response can be recorded.

Since the subject remains unaware of the stimulus, he or she experiences guessing rather than seeing. This is true not only for spatial location of stimuli but also for more intricate properties like shape or size (in this case the forced choice procedure is also used, the subject being asked to show on cards which of two patterns has been flashed within his scotoma). For details see Perenin and Jeannerod (1979). In other words, these patients are able to locate visually objects which they do not see. Furthermore, they may eventually be able to detect intrinsic properties in objects of which they are totally unaware. This is an example of the functioning of discrete neural mechanisms subserving discrete behavioral productions. The lesion artificially splits behavior into modular compartments and isolates segmental operations which normally cannot be dissociated.

At this point, the relevance of the concept of 'mental organs' (Chomsky, 1980) and of 'cognitive channels' to cognitive functioning, has to be ciscussed. These two terms have been taken here as equivalent. I consider the channel as a distributed neural ensemble bearing a particular transíer function, i.e., receiving information from the external world or from nther parts of the brain and releasing a predictable production. By this definiti on mental 
production is not considered as autonomous with respect to other productions of neural activity. It is only the input-output relationships which determine the specificity of a given channel, not the internal arrangement of its connections, which can be suspected to be similar to those of any other channel. In other words, the channel concept would definitely depart from current conceptions of mental operators elaborating their production from the inside, due to a particular 'genius' of theif internal structure (for example, see Popper ind Eccles, 1977).

The argument underlying this discussion is that attributirs to the mind structural properties which are not operational for other aspects of behavior will unavoidably result in a revival of the concept of autonomy of the 'mental level'. The mental-level concept implies the existence of some undefined 'higher level' of brain activity subserving cognitive 'functions'. Such an entity, however, has only a small chance of finding a precise embodiment in neurological terms, except that of a crude 'localization' in a given brain area. The notion of a mental level only concedes that cognition may have 'something to do' with neural mechanisms and that once a certain degree of complexity of a neural ensemble has been attained a new set of properties could emerge from that ensemble. In other words, although the mental level would admittedly be brain-dependent, it would not be reduced to the sum of simple netironal operations like those which are currently described for simpler levels of activity. The problem here is not with the lack of a satisfactory description of neuronal operations or connections which could account for cognitive responses (though this may be a real problem): it is rather with the incompatibility of the levels of explanation postulated $f . \quad$ leural functioning on one hand and for mental functioning on the other. In this context, neural mechan isms would represent but a useless ornament for cognition, and the whole concept of mental level would fail to reach a heuristic value, due to its dualistic and teleological underlying nature.

\section{References}

Chomsky, N. (1980) Rules and representations. Behav. Bruin Sci., 3, 1-63.

Jeannerod, M. (1981) Intersegmental coordination during reaching at natural visual objects. In J. Long and A. Baddeley (eds.), Attention and Performance $I X$. Hillsciale, NJ, Erlbaum, pp 153-168. Perenin, M. T., and Jeannerod, M. (1979) Subcortical vision in man. Trends Neurosci., 2, 204-207. Popper, K., and Eccles, J. C. (1977) The Self and its Brain. Berlin, Springer International.

Posner, M. I., Pea, R., and Volpe, B. (In press) Cognitive Neurscience, Toward ti Science of Synthesis. 